\title{
Controlled Activation of Substrate Templating in Molecular Self- Assembly by Deprotonation
}

\author{
Markus Kittelmann, ${ }^{\dagger}$ Markus Nimmrich, ${ }^{\dagger}$ Julia L. Neff, ${ }^{\dagger}$ Philipp Rahe, ${ }^{\dagger \dagger}$ Wojciech Greń, ${ }^{\S}$ Xavier Bouju, ${ }^{\S}$ \\ André Gourdon, ${ }^{\S}$ and Angelika Kühnle*, ${ }^{\dagger}$
${ }^{\dagger}$ Institut für Physikalische Chemie, Johannes Gutenberg-Universität Mainz, Duesbergweg 10-14, 55128 Mainz, Germany
${ }^{\S}$ Centre d'élaboration de matériaux et d'études structurale (CEMES), UPR 8011 CNRS, Nanosciences Group, 29 Rue J. Marvig, P.O. Box 94347, 31055 Toulouse, France

\begin{abstract}
Templated assembly of organic molecules constitutes a promising approach for fabricating functional nanostructures at surfaces with molecular-scale control. Using the substrate template for steering the adsorbate growth enables creating a rich variety of molecular structures by tuning the subtle balance of intermolecular and molecule-surface interactions. On insulating surfaces, however, surface templating is largely absent due to the comparatively weak molecule-surface interactions compared to metallic substrates. Here, we demonstrate the activation of substrate templating in molecular self-assembly on a bulk insulator by controlled deprotonation of the adsorbed molecules upon annealing. Upon deposition of 4-iodobenzoic acid onto the natural cleavage plane of calcite held at room temperature, high molecular mobility is observed, indicating a small diffusion barrier. Molecular islands only nucleate at step edges. These islands show no commensurability with the underlying substrate, clearly indicating the absence of surface templating. Upon annealing the substrate, the molecules undergo a transition from the protonated to the deprotonated state. In the deprotonated state, the molecules adopt a well-defined adsorption position, resulting in a distinctly different, substrate-templated molecular structure that is stable at room temperature. Our work, thus, demonstrates the controlled activation of substrate templating by changing the molecule-surface interaction upon annealing.

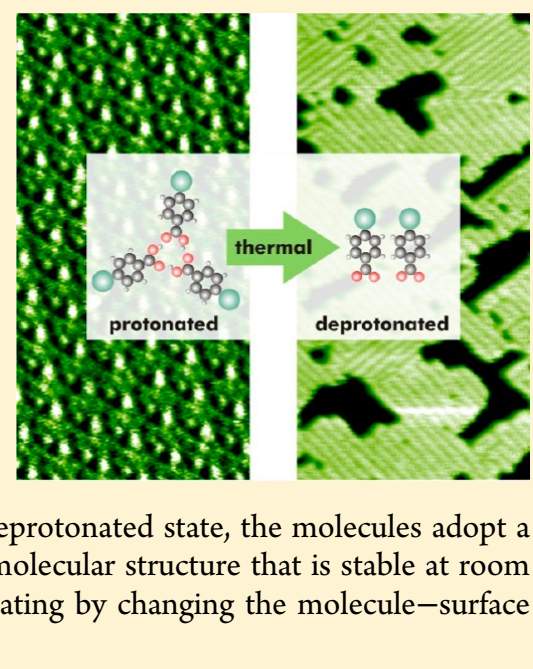

\begin{abstract}
upramolecular assembly based on reversible interactions is $\checkmark$ known as an extremely versatile synthesis tool for creating complex functional structures from molecular building blocks. ${ }^{1}$ Molecular self-assembly at surfaces benefits from the templating effect of the underlying substrate, which greatly enriches the structural variety by controlling the balance between intermolecular and molecule-surface interactions. ${ }^{2}$ In the past decades, an impressive variety of self-assembled molecular structures have been obtained upon adsorption of organic molecules onto metallic substrates under vacuum conditions, ${ }^{3-7}$ and to less extent onto semiconducting surfaces. ${ }^{8-11}$ However, from an application point of view, it is highly important to extend the range of substrates to dielectric materials in order to benefit from a larger materials basis. In particular, emerging nanotechnology applications such as molecular electronics require electrical decoupling of the molecular structures from the supporting surface. Pivotal experiments investigating molecular self-assembly on dielectric surfaces or thin films have, however, disclosed fundamental challenges, which are related to the weak molecule-surface binding. ${ }^{12-14}$ Furthermore, due to the absence of sufficient substrate templating, the resulting molecular structures are frequently governed by intermolecular interactions alone. This often results in molecular bulk crystal formation ${ }^{12}$ without the possibility to deliberately steer structural variety.
\end{abstract}

Thus, to transfer the full potential of molecular self-assembly to insulating substrates, strategies to induce substrate templating have to be explored. ${ }^{15}$ Recently, the electrostatic anchoring of polar molecules onto ionic crystal surfaces such as $\mathrm{CaF}_{2}(111)^{16}$ and $\mathrm{KBr}(001)^{17}$ has been explored to increase substrate templating. Moreover, the natural cleavage plane of calcite, $\mathrm{CaCO}_{3}(10.4)$, has been identified as a suitable substrate surface for molecular self-assembly at room temperature due to its comparatively high surface energy. ${ }^{18}$ In particular, carboxylic acid moieties have been exploited for creating substratetemplated structures that deviate substantially from the respective bulk structure. ${ }^{19}$ An interesting situation is obtained in the case of biphenyl-4,4'-dicarboxylic acid on $\mathrm{CaCO}_{3}(10.4)$, which shows the coexistence of two distinctly different molecular structures. One phase is governed by the underlying substrate periodicity and can be readily explained by the excellent size match of the molecular $\mathrm{COOH}-\mathrm{COOH}$ spacing and the calcite carbonate distance along the [01.0] surface direction. The second phase, in contrast, closely resembles the packing motif of the molecular bulk structure. ${ }^{20}$

When anchoring carboxylic acid moieties onto the (10.4) surface of calcite, the charge state of the acid group plays a

Received: August 29, 2013

Revised: October 16, 2013

Published: October 17, 2013 
(a)

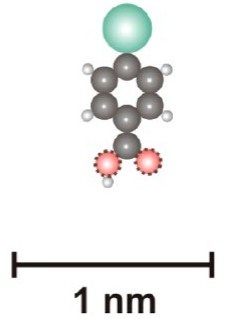

(b)

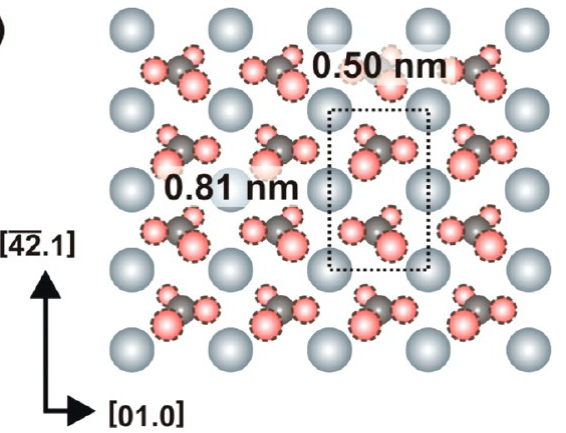

Figure 1. Model of the (a) 4-iodobenzoic acid (4IBA) molecule and (b) the $\mathrm{CaCO}_{3}(10.4)$ surface. The scale bar applies to both panels. The $\mathrm{CaCO}_{3}(10.4)$ surface has a rectangular unit cell of size $0.50 \times 0.81 \mathrm{~nm}^{2}$ consisting of two carbonate groups and two calcium ions. The carbonate groups are rotated such that one oxygen atom lies above, one in, and one below the plane spanned by the calcium ions. The different orientation of the two carbonate groups leads to a characteristic zigzag pattern of the topmost oxygen atoms. ${ }^{21}$

crucial role: In the protonated state, the molecules can form intermolecular hydrogen bonds as well as hydrogen bonds toward the calcite carbonate group. Protonated molecules are observed to lie flat on the surface to accommodate intermolecular hydrogen bonding. In the deprotonated state, on the other hand, the negatively charged carboxylate group anchors toward the surface calcium cations, and the molecules are observed to often stand upright on the surface. ${ }^{22,23}$ In a recent study, carboxylic acids with a low $\mathrm{p} K_{\mathrm{a}}$ value have been found to deprotonate already at room temperature while carboxylic acids with a $\mathrm{p} K_{\mathrm{a}}$ value higher than $\approx 3$ stay intact on calcite. $^{22}$ In the latter case, deprotonation can be induced by annealing the calcite substrate. ${ }^{23}$ Because the deprotonation of the molecule on calcite constitutes an acid-base reaction, we expect the proton to be linked to a surface carbonate group, forming hydrogen carbonate.

Here, we benefit from the distinct change in the moleculesurface interaction by controlled deprotonation of 4-iodobenzoic acid (4IBA). When deposited onto calcite held at room temperature, intermolecular interactions clearly dominate over the molecule-surface interaction, resulting in the formation of an ordered structure that exhibits no epitaxial relationship to the underlying calcite lattice. Interestingly, substrate templating can be activated by changing the molecule-surface interaction when annealing the substrate. Upon annealing, two different molecular phases coexist that are both governed by the molecule-surface interaction. This work illustrates that substrate templating can be activated deliberately by inducing deprotonation.

\section{RESULTS AND DISCUSSION}

Upon deposition of 4IBA onto calcite(10.4) held at room temperature (see Figure 1), large areas of the surface are found to remain unchanged except for a somewhat higher defect density. Molecules are exclusively found in troughs formed by step edges, nucleating into extended, highly ordered islands with an apparent height of approximately $0.5 \mathrm{~nm}$. Noncontact atomic force microscopy (NC-AFM) images of such islands are given in Figure 2a,b, showing two straight step edges running from the upper to the lower part of the images (marked by dashed lines). The troughs formed by the step edges are filled by molecular islands (bright area). From consideration of the molecular bulk structure and on the basis of our previous findings, ${ }^{20,23}$ a dimerization of the molecules via the carboxylic acid groups might be expected. However, the hexagonal structure observed here suggests molecular trimers rather than dimers. Our density functional theory (DFT) calculations show that a 4IBA trimer in the gas phase is only $0.06 \mathrm{eV} /$ molecule less stable than the dimer (see Methods for methodology details on calculations). This is different on the surface as our force field calculations indicate that although dimers are more stable when the molecules are isolated, the presence of the surface favors trimers instead of dimers. The existence of extended islands readily indicates that individual molecules, dimers, or trimers are mobile on calcite held at room temperature. The calculated potential energy surface for a trimer is shown in Figure 3. It reveals that the maximum diffusion barrier is about $0.3 \mathrm{eV}$. Similar calculations for the dimer structure resulted in a barrier of $0.4 \mathrm{eV}$, which indicates that the molecules can diffuse at room temperature (RT) in both dimeric and trimeric forms. This is in perfect agreement with the experimental finding of extended islands at room temperature. A zoom into the molecular island is given in the drift-corrected images shown in Figure 2c,d. In these images, the molecular structure imaged on two different islands is displayed. The molecules self-assemble into a hexagonal structure with only few lattice defects. Interestingly, the molecular pattern lacks an epitaxial relationship to the underlying calcite(10.4) surface, but the molecular structure seems to follow the step edge orientation as demonstrated in Figure $2 \mathrm{c}, \mathrm{d}$ by the dashed lines. Moreover, the overlayer lattice periodicity of $1.30 \pm 0.05 \mathrm{~nm}$ is incommensurate with the calcite lattice along the respective directions. These findings clearly indicate that the molecular layer is largely unperturbed by the calcite lattice, although the surface induces trimerization instead of dimerization. However, due to the missing epitaxial relationship and based on the lattice incommensurability, we expect the intermolecular interaction to govern the structure formation.

Provided that the interaction with the surface is small, we can evaluate the structure of the monolayer by running the molecular mechanics simulations in two dimensions and in the absence of the substrate. We consider a two-dimensional crystal structure of molecular trimers with the positions of the terminal hydrogen and oxygen atoms of the $\mathrm{COOH}$ groups constrained in the plane, while the remaining atoms are free to move. To represent the periodicity of the molecular structure, we apply periodic boundary conditions. The lowest energy structure is calculated with constant pressure minimization. The obtained molecular structure exhibits a hexagonal symmetry with a lattice parameter of $1.31 \mathrm{~nm}$ (Figure $2 \mathrm{e}$ ), which is very close to the average distance between the bright features 

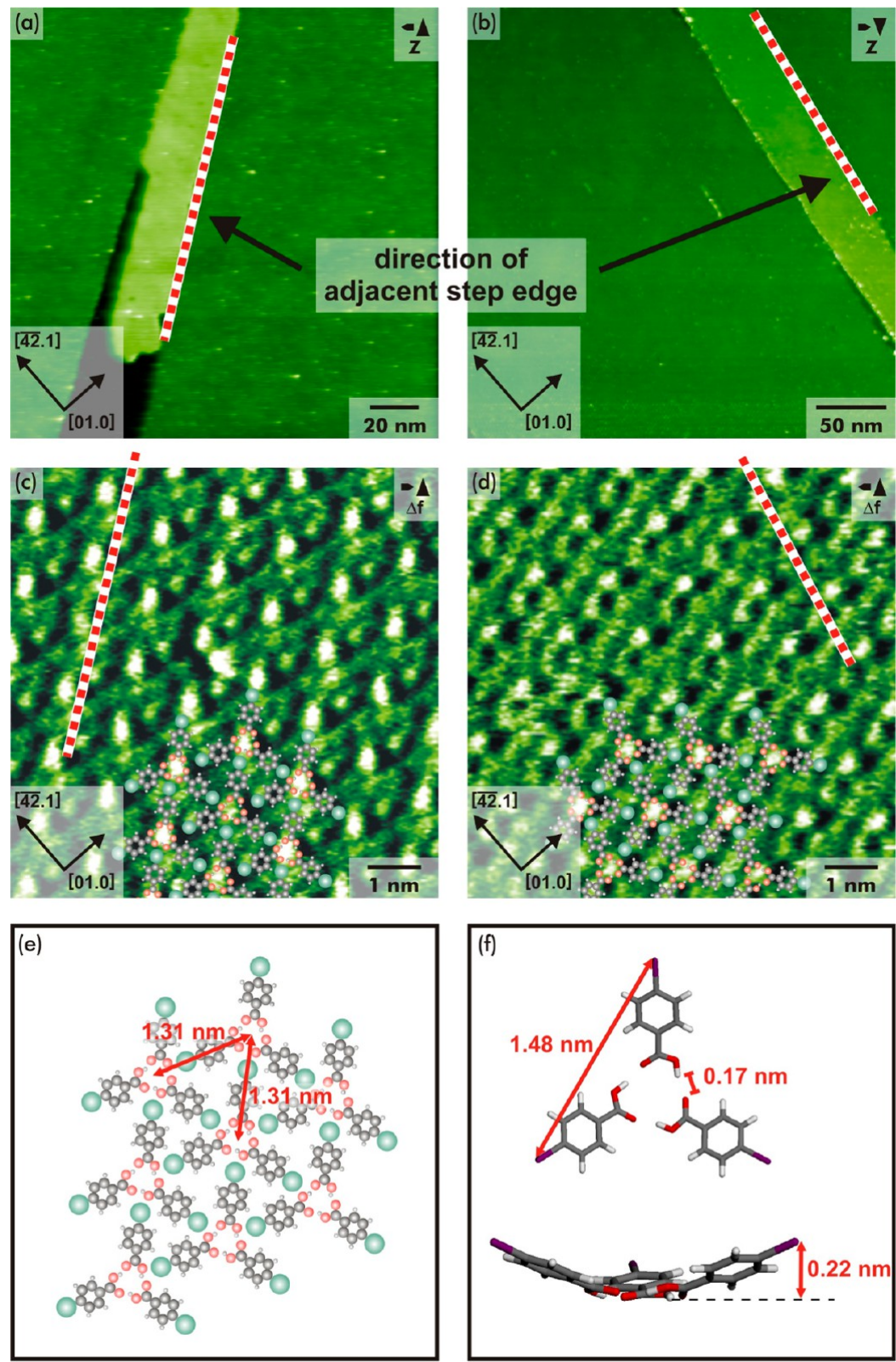

Figure 2. Molecular structures of 4IBA after deposition onto the natural cleavage plane of calcite held at room temperature. (a and b) Overview NCAFM images, revealing a surface that is largely free of molecules. Molecular islands only exist within surface troughs formed by two step edges (one of each is marked by dashed lines in each image). ( $c$ and d) High-resolution images taken on the two different islands (shown in panels a and b), illustrating the same internal order which lacks a fixed epitaxial relationship to the underlying substrate lattice. The respective step edge direction is again indicated by dashed lines. (e) Structural model for the alignment within the island in panel c, composed of the 4IBA trimers, calculated by twodimensional lattice minimization. (f) Geometry of the trimeric building unit in top and side views.

obtained in the experimental image. In this configuration, the carboxylic acid groups point to each other, allowing for hydrogen bond formation in a three-membered ring. The iodine atoms protrude by $0.22 \mathrm{~nm}$ out of the initial plane (see Figure $2 \mathrm{f}$ ). Such a structure is in very good agreement with the experimental results, assuming that the bright features in the images originate from the centers of the trimers.

When annealing the 4IBA-covered calcite substrate to $495 \mathrm{~K}$, the molecular structure changes significantly. After annealing, the entire surface is covered by two perpendicular striped structures (referred to as A and B) with an apparent height of approximately $0.8 \mathrm{~nm}$ (Figure $4 \mathrm{a}$ ). The increased apparent height suggests that the molecules now arrange in a more upright fashion as compared to the room-temperature islands. ${ }^{24}$
The change in coverage can be understood from the fact that weakly bound molecules that are not trapped in troughs diffuse rapidly on the surface. Rapidly diffusing molecules are known to be difficult to detect by NC-AFM. Thus, we propose that we do not image the diffusing molecules before annealing.

By making use of the Kelvin probe force microscopy (KPFM) technique, ${ }^{25}$ we can detect changes in the local contact potential difference. The voltage applied to the tip differs by approximately $-2 \mathrm{~V}$ above the striped structures in comparison to the bare calcite surface. This shift can be readily understood by considering the change in the charge state upon deprotonation. After deprotonation, the molecules are negatively charged. On the basis of this and our previous findings, we ascribe this structural change to a deprotonation step, which 
(a)

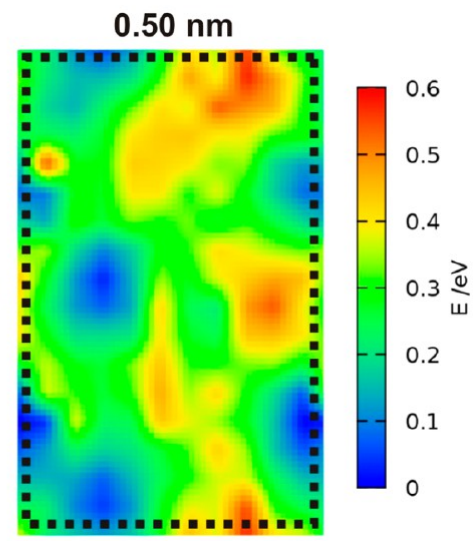

(b)

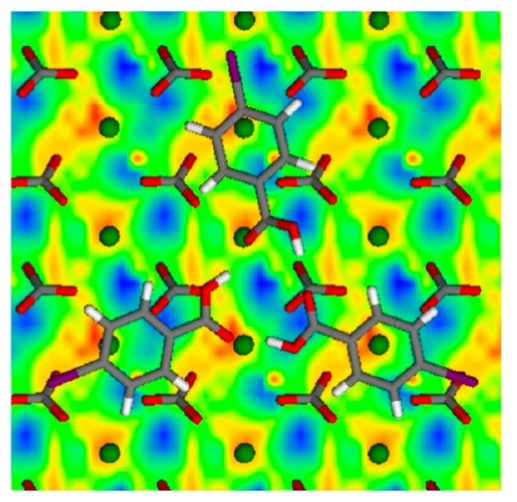

Figure 3. Calculated potential energy surface for a 4IBA trimer on the calcite(10.4) surface. (a) Calculations for one unit cell reveal four minima separated by barriers of about $0.4 \mathrm{eV}$, which indicates that trimer molecules diffuse on the surface at room temperature. (b) Superimposing the substrate structure on the energy surface shows that two of the lowest energy sites are between carbonate ions while the other two are between a pair of calcium ions and a carbonate ion.

requires annealing in the case of 4IBA due to its comparatively high $\mathrm{p} K_{\mathrm{a}}$ value of $4.02 .^{22,23}$ In the $\mathrm{A}$ phase, the stripes are oriented along the $[\overline{42} 1]$ direction, while in the $B$ phase, the stripes are running along the [01.0] direction. A zoom into the A phase (Figure $4 \mathrm{~b}$ ) discloses a dotted pattern, where each dot fits in size with an upright-standing molecule. The zoom in Figure $4 \mathrm{~b}$ indicates that the stripes originate from a moiré pattern. Drift-corrected high-resolution images of the A and B phases are shown in Figure 4c,d, respectively.

Phase A is composed of protrusions with a repeat distance of approximately $a_{x}=0.58 \pm 0.05 \mathrm{~nm}$ in the [01.0] direction and $a_{y}=0.79 \pm 0.05 \mathrm{~nm}$ in the $[\overline{42} 1]$ direction. In the $[\overline{42} 1]$ direction, the molecules adopt the substrate periodicity of 0.81 $\mathrm{nm}$. Along the [01.0] direction, however, seven molecules are spaced atop eight calcite repeat distances, resulting in a $(8 \times 1)$ superstructure $(7 \times 0.58 \mathrm{~nm}=4.06 \mathrm{~nm}$ equals $8 \times 0.50 \mathrm{~nm}=$ $4.00 \mathrm{~nm}$; see Figure 4e). The difference in lattice spacing along the [01.0] direction readily explains a moiré pattern with stripes running perpendicular to the [01.0] direction as evident in Figure $4 \mathrm{~b}$.

Phase B is formed by dots with a periodicity of approximately $b_{x}=1.01 \pm 0.05 \mathrm{~nm}$ along the [01.0] direction, which equals two times the calcite unit cell dimension in this direction. Along the $[\overline{42} 1]$ direction, however, the molecular pattern shows a distance of approximately $b_{y}=0.57 \pm 0.05 \mathrm{~nm}$, which is different from the substrate periodicity. Along this direction, seven molecules are spaced along five calcite unit cell repeat distances $(7 \times 0.57 \mathrm{~nm}=3.99 \mathrm{~nm}$ equals $5 \times 0.81 \mathrm{~nm}=4.05$ $\mathrm{nm})$, resulting in a $(2 \times 5)$ superstructure (Figure $4 \mathrm{e})$. Again, the different periodicity of the substrate and the molecular pattern gives rise to a moiré pattern, which is for phase $B$ perpendicular to the $[\overline{42} 1]$ direction.

The existence of the moire pattern clearly indicates that the molecular structures are steered by the underlying substrate in one direction (the $[\overline{42} 1]$ direction for phase $\mathrm{A}$ and the [01.0] direction for phase $\mathrm{B}$ ), while the distance in the other direction is influenced by the intermolecular interaction. Interestingly, the spacing of phase A along the [01.0] direction is similar to the spacing of phase $\mathrm{B}$ along the $[\overline{42} 1]$ direction, suggesting that this spacing is dominated by the intermolecular interaction. This can be understood by considering a tilted arrangement of the molecules as given in Figure 4f,g, in which the iodine atoms are placed above the center of the aromatic ring of the neighboring molecule. ${ }^{26}$ This configuration is in accordance with upright standing deprotonated molecules anchoring toward the surface with the negatively charged carboxylate groups that bind to the surface calcium cations. Thus, by deprotonating the molecules, the molecule-surface interaction is greatly increased and substrate-templated structures emerge.

\section{CONCLUSION}

In conclusion, the controlled activation of substrate templating is demonstrated by inducing a deprotonation reaction of 4IBA on the natural cleavage plane of calcite. When depositing 4IBA onto calcite held at room temperature, the molecules are found to be highly mobile on the surface. The molecules exclusively nucleate at step edges and arrange themselves into an ordered array of trimers with hydrogen bond formation between the carboxylic acid groups. The latter finding gives strong evidence for the fact that the molecules remain protonated in this structure. Most interestingly, these islands lack an epitaxial relationship to the underlying calcite lattice, clearly demonstrating the absence of substrate templating. This situation is changed reproducibly upon annealing the substrate to $495 \mathrm{~K}$, which results in molecule deprotonation. After deprotonation, two different phases are formed that are distinctly different from the former hydrogen-bonded structure. Now, the molecules stand upright on the surface, with the negatively charged carboxylate groups anchoring toward the surface calcium cations. This substrate templating effect is clearly reflected in the molecular ordering that now follows the substrate periodicity in one direction (the [ $\overline{42} 1]$ direction for phase $A$ and the [01.0] direction for $B$, respectively). Perpendicular to this direction, a moiré pattern is observed, which is governed by the optimum packing of the aromatic rings. This study demonstrates that molecular deprotonation can be successfully exploited to deliberately activate substrate templating in molecular self-assembly.

\section{METHODS}

Sample preparation and noncontact atomic force microscopy (NC-AFM) experiments were carried out under ultrahighvacuum (UHV) conditions. Calcite crystals of optical quality were purchased from Korth Kristalle $\mathrm{GmbH}$ (Altenholz, Germany) and cut mechanically to fit into the sample holder. After introduction into the UHV system, each crystal was 

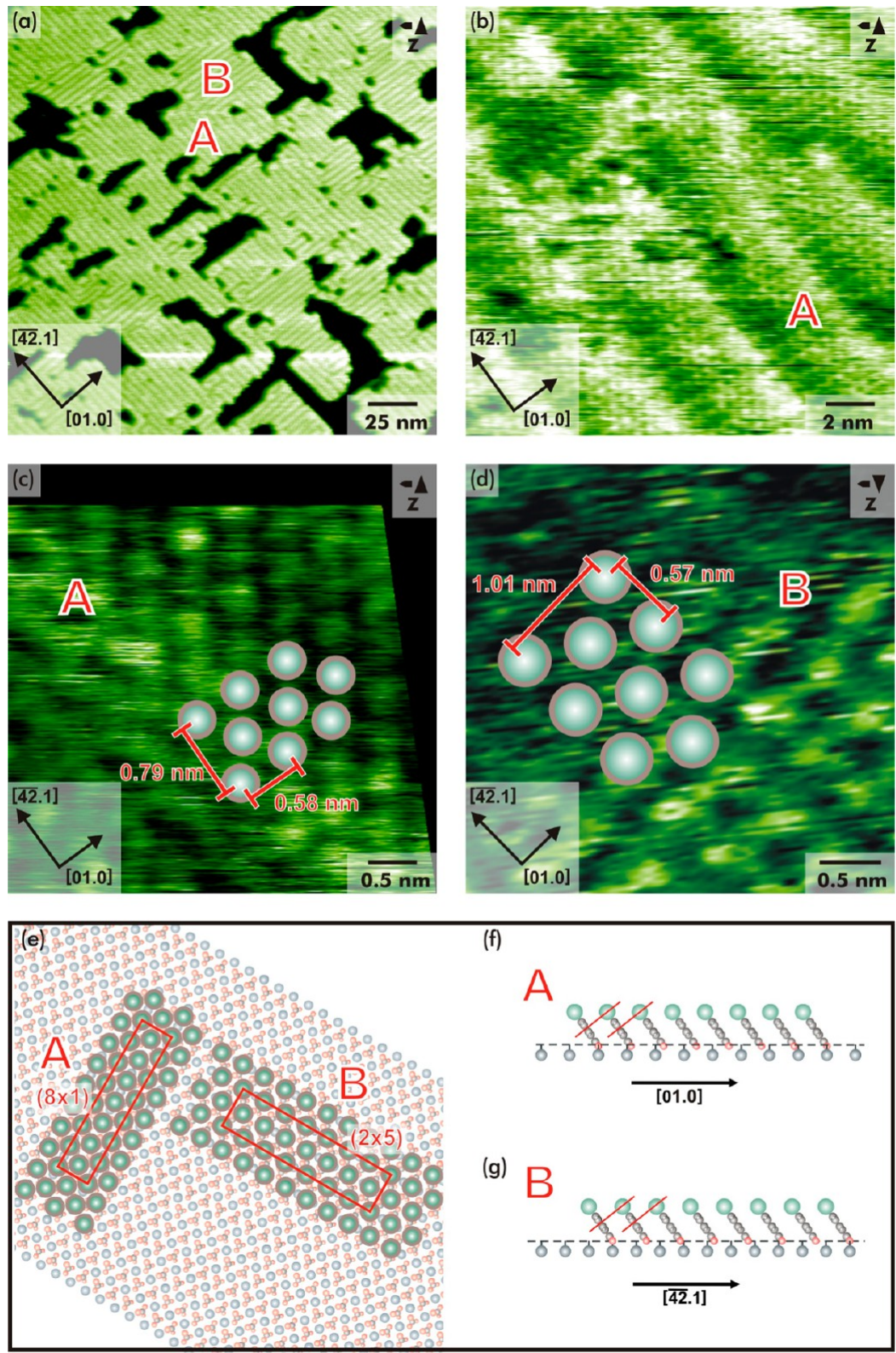

Figure 4. Molecular structures after annealing the substrate to $495 \mathrm{~K}$. (a) Overview NC-AFM image revealing two striped phases named A and B. (b) Zoom into the A phase, indicating that the stripes originate from a moire pattern. (c) Drift-corrected high-resolution NC-AFM image of the A phase, showing a centered rectangular packing that corresponds to a $(8 \times 1)$ superstructure. The black area arises from the drift correction procedure. (d) Drift-corrected high-resolution NC-AFM image of the B phase, showing a centered rectangular packing that corresponds to a $(2 \times 5)$ superstructure. (e) Top-view model illustrating the arrangement of the molecules within the two structures. (f) Side view along the [01.0] direction of the A phase, illustrating the tilted orientation of the molecules and the resulting alignment of the iodine atoms and the aromatic rings. The optimum molecular packing results in a moiré pattern with seven molecules arranged along a distance of eight lattice repeat units. (g) Corresponding view of the $\mathrm{B}$ phase along the $[\overline{42} 1]$ direction. In this direction, seven molecules are spaced along five lattice repeat units.

freshly cleaved ${ }^{27}$ and annealed for about $1.5 \mathrm{~h}$ to a maximum temperature of about $450 \mathrm{~K}$ to remove surface charges. A pristine crystal was used for each molecule deposition. The surface orientation was determined for each crystal by measuring the unit cell dimensions in images that were carefully corrected for thermal drift. ${ }^{28}$ The NC-AFM experiments were performed with an atomic force microscope (VT AFM 25 from Omicron, Taunusstein, Germany) operated in the frequency modulated (FM) mode. ${ }^{29}$ In this mode, the change $\Delta \mathrm{f}$ of the cantilever's resonance frequency upon tipsample interaction is the main measurement signal. This signal is related to the tip-sample interaction force. ${ }^{30,31}$ The frequency shift $\Delta f$ was measured using a phase-locked loop controller (easyPLL plus from Nanosurf, Liestal, Switzerland). FM-KPFM measurements are performed by applying an alternating current (AC) voltage to the tip (frequency, 1 $\mathrm{kHz}$; amplitude, $2.0 \mathrm{~V}$ ) and compensating for the resulting electrostatic force with an offset direct current (DC) voltage applied to the tip (Kelvin signal) using a digital lock-in amplifier with built-in feedback loop (HF2 from Zurich Instruments, Zurich, Switzerland). All NC-AFM data were analyzed and processed using the open source software Gwyddion. ${ }^{32}$ Standard Si cantilevers (type PPP-NCH from Nanosensors, Neuchâtel, Switzerland) with resonance frequencies around 300 
$\mathrm{kHz}$ were excited to amplitudes around $10 \mathrm{~nm}$. All tips were initially bombarded with $\mathrm{Ar}^{+}$ions to remove contaminants and the oxide layer. The image channel as well as fast and slow scan directions are given in the upper right corner of each image. 4IBA molecules were purchased from Sigma Aldrich (98\% purity). The deposition was performed from a heated glass crucible. Prior to the experiments shown here, the deposition rate was determined with a quartz crystal microbalance. A temperature of $365 \mathrm{~K}$ corresponds to a sublimation rate of about $0.04 \mathrm{ML} / \mathrm{min}$.

The modeled surface was prepared and minimized with METADISE code, ${ }^{33}$ while DLPOLY ${ }^{34}$ was used to study the interface between the molecules and the surface. We employed the force field parameters developed by Pavese et al. ${ }^{35}$ for calcite and Freeman et al. ${ }^{36}$ for interactions between the 4IBA molecules and the surface. The intra- and intermolecular interactions in 4IBA were described by the DREIDING model, $^{37}$ and parameters for the $\mathrm{COOH}$ group were based on the model of Roszak et al. ${ }^{38}$ The parameters were verified by running DFT calculations using SIESTA $\operatorname{code}^{39}$ with PBE functional, ${ }^{40}$ double- $\zeta$ polarized basis set (DZP), and norm conserving Troullier-Martins pseudopotentials. ${ }^{41}$ The $3 p$ electrons for $\mathrm{Ca}$ were explicitly included in the valence. The partial charges for 4IBA molecule were obtained by RESP method $^{42}$ using Quickstep/CP2K code. ${ }^{43}$ The potential energy surface for a dimer and trimer translation was calculated by running a series of constrained minimizations, where the center of mass of the cluster was fixed in the $X Y$ plane but allowed to relax in the $Z$ direction, and by allowing a rotation. The barriers were refined using the nudged elastic band (NEB) method. ${ }^{44}$ To obtain a better agreement between the force field and the DFT structures, the parameters $\varepsilon$ and $r_{0}$, describing the hydrogen bonds between 4IBA molecules, were adjusted to $\varepsilon=$ $0.08 \mathrm{eV}$ and $r_{0}=1.7 \AA$. The hydrogen bond between the 4IBA monomer and the surface was described by the Lennard-Jones 9-6 potential with parameters $\varepsilon=0.05 \mathrm{eV}$ and $r_{0}=1.9 \AA$.

\section{AUTHOR INFORMATION}

\section{Corresponding Author}

*E-mail: kuehnle@uni-mainz.de. Tel.: +49 61313923930.

\section{Present Address}

${ }^{\ddagger}$ Department of Physics and Astronomy, The University of Utah, 115 South 1400 East, Salt Lake City, UT 84112, USA.

\section{Notes}

The authors declare no competing financial interest.

\section{ACKNOWLEDGMENTS}

Financial support from the joint German Research Foundation and Agence Nationale de la Recherche (DFG/ANR) project "In Situ Chemistry of Molecular Assemblies on Dielectric Surfaces" (ICMADS) through Grants KU 1980/5-1 and ANR11-INTB-1007 is gratefully acknowledged. Part of this work was performed using High Performance Computing resources from the Calcul en Pyrénées (CALMIP) facilities (Grant No. 2011-[P0832]).

\section{REFERENCES}

(1) Lehn, J.-M. Toward Self-Organization and Complex Matter. Science 2002, 295, 2400-2403.

(2) Barth, J. V. Molecular Architectonic on Metal Surfaces. Annu. Rev. Phys. Chem. 2007, 58, 375-407.
(3) Rosei, F.; Schunack, M.; Jiang, P.; Gourdon, A.; Lægsgaard, E.; Stensgaard, I.; Joachim, C.; Besenbacher, F. Organic Molecules Acting as Templates on Metal Surfaces. Science 2002, 296, 328-331.

(4) Pawin, G.; Wong, K. L.; Kwon, K. Y.; Bartels, L. A Homomolecular Porous Network at a $\mathrm{Cu}(111)$. Surf. Sci. 2006, 313, 961-962.

(5) Xu, W.; Dong, M.; Gersen, H.; Rauls, E.; Vazquez-Campos, S.; Crego-Calama, M.; Reinhoudt, D. N.; Stensgaard, I.; Laegsgaard, E.; Linderoth, T. R; et al. Cyanuric Acid and Melamine on $\mathrm{Au}(111)$ : Structure and Energetics of Hydrogen-Bonded Networks. Small 2007, $3,854-858$.

(6) Écija, D.; Seufert, K.; Heim, D.; Auwärter, W.; Aurisicchio, C.; Fabbro, C.; Bonifazi, D.; Barth, J. V. Hierarchic Self-Assembly of Nanoporous Chiral Networks with Conformationally Flexible Porphyrins. ACS Nano 2010, 4, 4936-4942.

(7) Guillermet, O.; Niemi, E.; Nagarajan, S.; Bouju, X.; Martrou, D.; Gourdon, A.; Gauthier, S. Self-Assembly of Fivefold-Symmetric Molecules on a Threefold-Symmetric Surface. Angew. Chem., Int. Ed. 2009, 48 (11), 1970-1973.

(8) Hamers, R. J.; Coulter, S. K.; Ellison, M. D.; Hovis, J. S.; Padowitz, D. F.; Schwartz, M. P.; Greenlief, C. M.; Russell, J. N. Cycloaddition Chemistry of Organic Molecules with Semiconductor Surfaces. Acc. Chem. Res. 2000, 33 (9), 617-624.

(9) Makoudi, Y.; Palmino, F.; Arab, M.; Duverger, E.; Chérioux, F. d. r. Complete Supramolecular Self-Assembled Adlayer on a Silicon Surface at Room Temperature. J. Am. Chem. Soc. 2008, 130, 66706671.

(10) Baris, B.; Luzet, V.; Duverger, E.; Sonnet, P.; Palmino, F.; Cherioux, F. Robust and Open Tailored Supramolecular Networks Controlled by the Template Effect of a Silicon Surface. Angew. Chem., Int. Ed. 2011, 50, 4094-4098.

(11) Baris, B.; Jeannoutot, J.; Luzet, V.; Palmino, F.; Rochefort, A.; Chérioux, F. Noncovalent Bicomponent Self-Assemblies on a Silicon Surface. ACS Nano 2012, 6, 6905-6911.

(12) Kunstmann, T.; Schlarb, A.; Fendrich, M.; Wagner, T.; Möller, R; Hoffmann, R. Dynamic Force Microscopy Study of 3,4,9,10Perylenetetracarboxylic Dianhydride on $\operatorname{KBr}(001)$. Phys. Rev. B: Condens. Matter Mater. Phys. 2005, 71, No. 121403.

(13) Bombis, C.; Kalashnyk, N.; Xu, W.; Lægsgaard, E.; Besenbacher, F.; Linderoth, T. R. Hydrogen-Bonded Molecular Networks of Melamine and Cyanuric Acid on Thin Films of $\mathrm{NaCl}$ on $\mathrm{Au}(111)$. Small 2009, 5 (19), 2177-2182.

(14) Such, B.; Trevethan, T.; Glatzel, T.; Kawai, S.; Zimmerli, L.; Meyer, E.; Shluger, A. L.; Amijs, C. H. M.; de Mendoza, P.; Echavarren, A. M. Functionalized Truxenes: Adsorption and Diffusion of Single Molecules on the $\operatorname{KBr}(001)$ Surface. ACS Nano 2010, 4, 3429-3439.

(15) Rahe, P.; Kittelmann, M.; Neff, J. L.; Nimmrich, M.; Reichling, M.; Maass, P.; Kühnle, A. Tuning Molecular Self-Assembly on Bulk Insulator Surfaces by Anchoring of the Organic Building Blocks. Adv. Mater. 2013, 25, 3948-3956.

(16) Schütte, J.; Bechstein, R.; Rohlfing, M.; Reichling, M.; Kühnle, A. Cooperative Mechanism for Anchoring Highly Polar Molecules at an Ionic Surface. Phys. Rev. B: Condens. Matter Mater. Phys. 2009, 80, 205421.

(17) Glatzel, T.; Zimmerli, L.; Kawai, S.; Meyer, E.; Fendt, L.-A.; Diederich, F. Oriented Growth of Porphyrin-Based Molecular Wires on Ionic Crystals Analysed by NC-AFM. Beilstein J. Nanotechnol. 2011, 2, 34-39.

(18) Rahe, P.; Lindner, R.; Kittelmann, M.; Nimmrich, M.; Kühnle, A. From Dewetting to Wetting Molecular Layers: C60 on $\mathrm{CaCO}_{3}(10 \overline{1} 4)$ as a Case Study. Phys. Chem. Chem. Phys. 2012, 14, 6544-6548.

(19) Rahe, P.; Nimmrich, M.; Kühnle, A. Substrate Templating upon Self-Assembly of Hydrogen-Bonded Molecular Networks on an Insulating Surface. Small 2012, 8, 2968-2968.

(20) Kittelmann, M.; Rahe, P.; Kühnle, A. Molecular Self-assembly on an Insulating Surface: Interplay between Substrate Templating and 
Intermolecular Interactions. J. Phys.: Condens. Matter 2012, 24, 354007-354013.

(21) Rahe, P.; Schütte, J.; Kühnle, A. NC-AFM Contrast Formation on the Calcite (1014) Surface. J. Phys.: Condens. Matter 2012, 24, 084006.

(22) Kittelmann, M.; Rahe, P.; Nimmrich, M.; Hauke, C. M.; Gourdon, A.; Kühnle, A. On-Surface Covalent Linking of Organic Building Blocks on a Bulk Insulator. ACS Nano 2011, 5, 8420-8425.

(23) Kittelmann, M.; Rahe, P.; Gourdon, A.; Kühnle, A. Direct Visualization of Molecule Deprotonation on an Insulating Surface. ACS Nano 2012, 6, 7406-7411.

(24) We stress, however, that height measurements need to be considered with care as force rather than topography is sensed in NCAFM.

(25) Zerweck, U.; Loppacher, C.; Otto, T.; Grafström, S.; Eng, L. M. Accuracy and Resolution Limits of Kelvin Probe Force Microscopy. Phys. Rev. B: Condens. Matter Mater. Phys. 2005, 71, No. 125424.

(26) Prasanna, M. D.; Guru Row, T. N. C-Halogen ‥ Interactions and Their Influence on Molecular Conformation and Crystal Packing: A Database Study. Cryst. Eng. 2000, 3, 135-154.

(27) Tröger, L.; Schütte, J.; Ostendorf, F.; Kühnle, A.; Reichling, M. Concept for Support and Cleavage of Brittle Crystals. Rev. Sci. Instrum. 2009, 80, 063703 .

(28) Rahe, P.; Bechstein, R.; Kühnle, A. Vertical and Lateral Drift Corrections of Scanning Probe Microscopy images. J. Vac. Sci. Technol, B: Microelectron. Nanometer Struct.-Process., Meas., Phenom. 2010, 28, C4E31-C4E38.

(29) Albrecht, T. R.; Grütter, P.; Horne, D.; Rugar, D. FrequencyModulation Detection Using High-Q Cantilevers for Enhanced Force Microscope Sensitivity. J. Appl. Phys. 1991, 69, 668-673.

(30) Giessibl, F. J. Advances in Atomic Force Microscopy. Rev. Mod. Phys. 2003, 75, 949-983.

(31) Giessibl, F. J. Forces and Frequency Shifts in Atomic-Resolution Dynamic-Force Microscopy. Phys. Rev. B: Condens. Matter Mater. Phys. 1997, 56, 16010-16015.

(32) Neučas, D.; Klapetek, P. Gwyddion: an Open-Source Software for SPM Data Analysis. Cent. Eur. J. Phys. 2012, 10, 181-188.

(33) Watson, G. W.; Kelsey, E. T.; de Leeuw, N. H.; Harris, D. J.; Parker, S. C. Atomistic Simulation of Dislocations, Surfaces and Interfaces in MgO. J. Chem. Soc., Faraday Trans. 1996, 92, 433-438.

(34) Smith, W.; Forester, T. R. DL_POLY_2: A General-Purpose Parallel Molecular Dynamics Simulation Package. J. Mol. Graph. 1996, 14 (3), 136-141.

(35) Pavese, A.; Catti, M.; Parker, S. C.; Wall, A. Modelling of the Thermal Dependence of Structural and Elastic Properties of Calcite, $\mathrm{CaCO}_{3}$. Phys. Chem. Miner. 1996, 23, 89-93.

(36) Freeman, C. L.; Harding, J. H.; Cooke, D. J.; Elliott, J. A.; Lardge, J. S.; Duffy, D. M. New Forcefields for Modeling Biomineralization Processes. J. Phys. Chem. C 2007, 111, 1194311951.

(37) Mayo, S. L.; Olafson, B. D.; Goddard, W. A. DREIDING: A Generic Force Field for Molecular Simulations. J. Phys. Chem. 1990, 94, 8897-8909.

(38) Roszak, S.; Gee, R. H.; Balasubramanian, K.; Fried, L. E. New Theoretical Insight into the Interactions and Properties of Formic Acid: Development of a Quantum-Based Pair Potential for Formic Acid. J. Chem. Phys. 2005, 123, No. 144702.

(39) José, M. S.; Emilio, A.; Julian, D. G.; Alberto, G.; Javier, J.; Pablo, O.; Daniel, S.-P. The SIESTA Method for ab Initio Order-N Materials Simulation. J. Phys.: Condens. Matter 2002, 14, 2745.

(40) Perdew, J. P.; Burke, K.; Ernzerhof, M. Generalized Gradient Approximation Made Simple. Phys. Rev. Lett. 1996, 77 (18), 38653868.

(41) Troullier, N.; Martins, J. L. Efficient Pseudopotentials for Plane Wave Calculations. Phys. Rev. B: Condens. Matter Mater. Phys. 1991, 43 (3), 1993-2006.

(42) Bayly, C. I.; Cieplak, P.; Cornell, W.; Kollman, P. A. A WellBehaved Electrostatic Potential Based Method Using Charge
Restraints for Deriving Atomic Charges: The RESP Model. J. Phys. Chem. 1993, 97, 10269-10280.

(43) VandeVondele, J.; Krack, M.; Mohamed, F.; Parrinello, M.; Chassaing, T.; Hutter, J. Quickstep: Fast and Accurate Density Functional Calculations Using a Mixed Gaussian and Plane Waves Approach. Comput. Phys. Commun. 2005, 167, 103-128.

(44) H. Jónsson, Mills, G.; Jacobsen, K. W. In Classical and Quantum Dynamics in Condensed Phase Simulations: Berne, B. J., Ciccotti, G., Coker, D. F., Eds.; World Scientific: Singapore, 1998; p 385. 